\title{
Radiculomedullary complications of cervical spinal manipulation
}

\author{
L Padua, R Padua, M LoMonaco and PA Tonali \\ Institute of Neurology and the Institute of Orthopaedics, Catholic University of Rome, Italy and the CSS Hospital \\ IRCCS San Giovanni Rotondo - Italy
}

\begin{abstract}
Spinal manipulation is commonly used by some therapists for the treatment of cervical pain. Flexion-extension of the cervical spine produces sliding movements of one vertebra over the one below it, which leads to physiological reduction in the antero-posterior diameter of the spinal canal. Spinal manipulation provokes movements that exceed the physiological limits of these articulations and thereby lead to a more significant reduction of the canal diameter. In patients with pre-existing stenosis of the canal or those with vertebral instability, these movements may cause (or aggravate) myelopathy. For this reason, a thorough neurological examination and cervical spine films should be considered mandatory in patients being considered for spinal manipulation. This report describes four patients with cervical myelopathy and/or radiculopathy caused or aggravated by spinal manipulation. In one patient, magnetic resonance scans before and after chiropractic treatment strongly suggests that the disc prolapse syndrome experienced by the patient was provoked by the spinal manipulation.
\end{abstract}

Keywords: myelopathy; radiculopathy; spinal manipulation; cervical disc protrusion

\section{Introduction}

Spinal manipulation is frequently used by some therapists to treat cervical pain. The cerebrovascular complications that can be provoked by such treatment of the cervical spine are well known, ${ }^{1,2}$ but there are relatively few reports of adverse effects involving the spinal cord and nerve roots. ${ }^{3-9}$ In the past 3 years we have observed four patients who developed problems of this type following manipulation of the cervical vertebrae. In one patient, magnetic reasonance scans (MRI) performed before and after manipulation strongly suggested that the chiropractic treatment was the cause of the cervical disc prolapse.

\section{Case reports}

\section{Case 1}

This 67-year-old male physician had suffered from recurrent non-radiating cervical pain for several years. Cervical spine X-rays, computed tomography (CT) and MRI (Figure la) demonstrated severe cervical arthrosis with central narrowing of the canal associated with features of vertebral instability (abnormal disc narrowing, translatory displacement). These symptoms had been periodically treated by a chiropractor with reportedly good results. Eight months later, the patient was treated for the same symptoms by a new chiropractor. During the first strong cervical manipula-

Correspondence: DR L. Padua tion by this therapist, the patient reportedly felt a sharp pain in his left arm followed by parasthesiae and severe weakness of this limb. The patient reported that he was unable to abduct his left arm, pick up a sheet of paper or hold a pen to write. Treatment was discontinued, but the symptoms persisted and one week later an MRI scan was performed, and this showed marked central narrowing of the cervical spinal canal and prolapse of the the C5-C6 and C6-C7 discs, which were not present in the MRI studies performed eight months previously (see Figure 1b).

Shortly thereafter he was seen by our staff. Neurological examination confirmed the presence of severe motor deficits in the left arm expecially of the intrinsic muscles of the hand. Superficial sensory deficit was observed in the $\mathrm{C} 7$ dermatones. No deficits or pathological signs were found in the trunk and other limbs. Electromyography revealed denervation of the muscles in the C5-T1 metameres. The damage was particularly severe at the C8-T1 level: fibrillation potentials and positive sharp waves at rest were noted in the deltoid, biceps brachii, extensor digitorum, I interosseus dorsalis and abductor pollicis. Voluntary movement of the latter two muscles was markedly reduced. The sensory nerve action potential registered at the left wrist after stimulation of the median nerve in the third digit was markedly reduced (1.3 microvolts), while all other sensory and motor studies were within normal limits. There was suggestion of post-ganglionic nerve injury at $\mathrm{C} 7$.

Somatosensory evoked potentials were normal. 


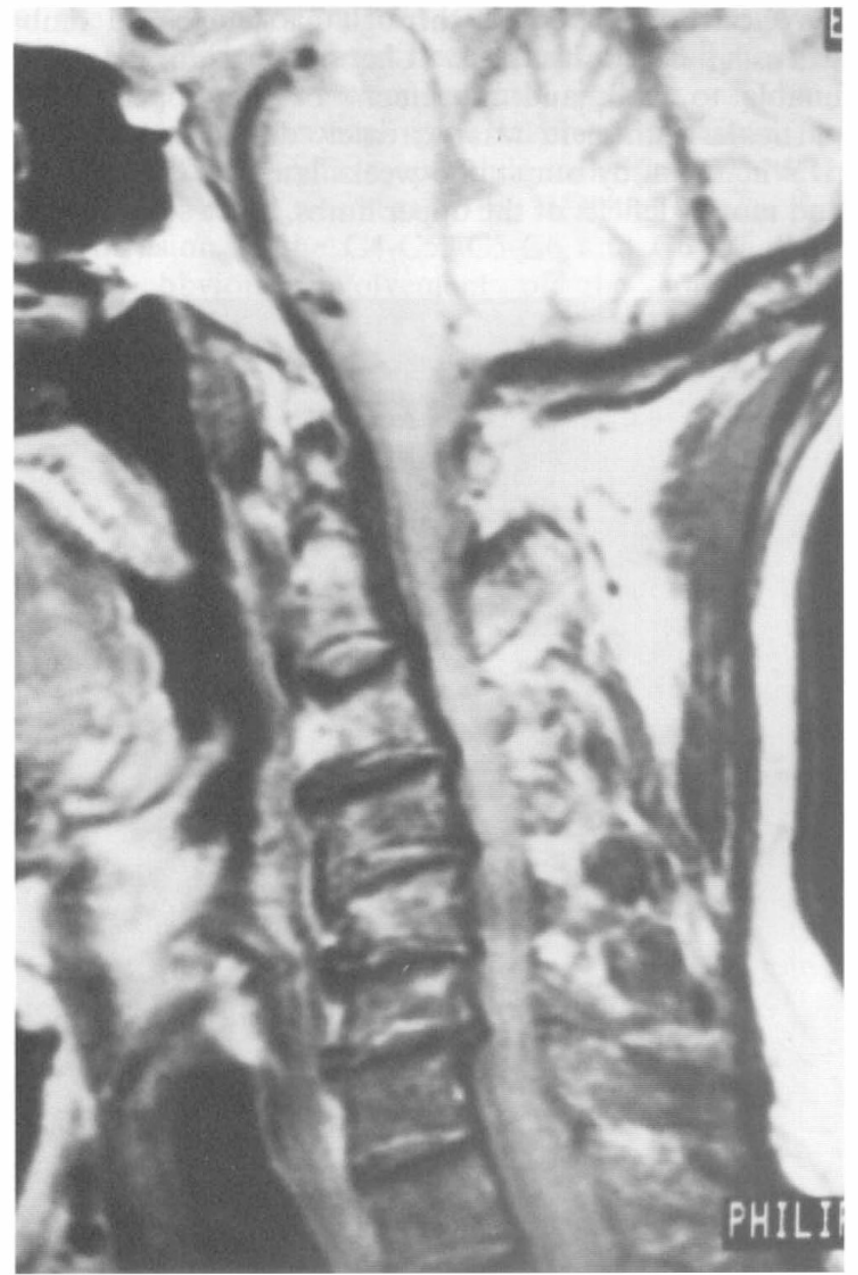

b

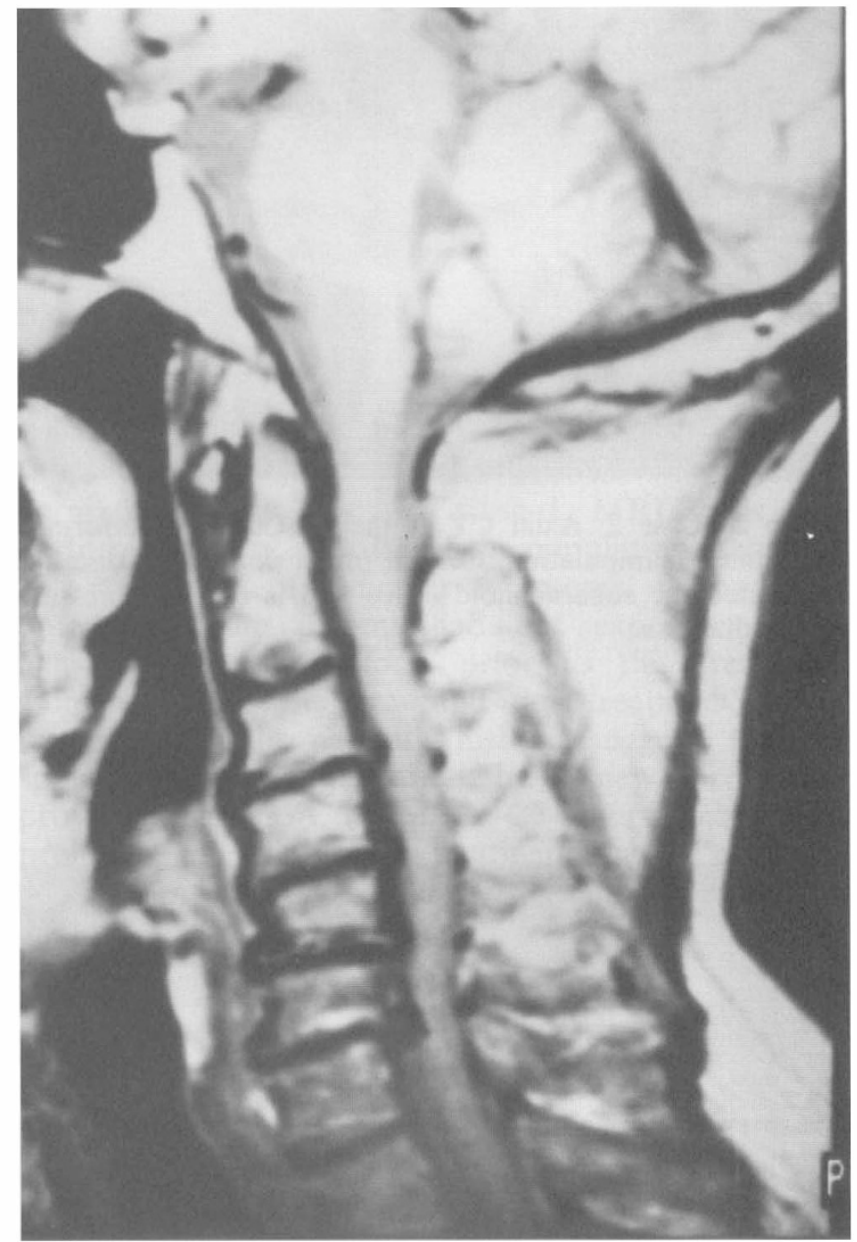

Figure 1 Case 1: Pre- (la) and post-manipulation (lb) sagittal T1-dependent MRIs. The post-treatment study shows prolapse of the C5-C6 and C6-C7 discs which was not present in the previous examination. Spondylosis at the C5-C6 level results in pressure on the ventral surface of the spinal cord. Note that the cuts (sequences) were the same in the pre and in the post treatment scans

The left-arm deficits have gradually improved without special treatment, and the patient's condition is currently stable. He has refused cervical spine surgery.

\section{Case 2}

This 60-year-old carpenter was referred to us because of parasthesiae in the hands and generalized limb weakness which had developed two hours after manipulation of his cervical spine for neck pain. This treatment had been continued in spite of the onset of these symptoms, but was discontinued shortly thereafter when the weakness and parasthesiae worsened.

Our neurological examination (1 week after the above episode) revealed weakness of both the upper and the lower limbs especially severe of the brachial biceps and triceps. The left patellar reflex was brisk and polykinetic and there was a Babinski sign on the left. The remainder of the examination was within normal limits. Computed tomography of the cervical spine revealed a bulky median $\mathrm{C} 4-\mathrm{C} 5$ disc herniation (Figure 2). MRI was not performed because the patient had suffered shrapnel injuries during the war. An electromyogram showed fibrillation potentials at rest and sharp positive waves within the C5 myotome (supraspinatus and deltoid). Somatosensory evoked potentials indicated mild cervical myelopathy. The patient was treated with a cervical collar. Progressive improvement was observed and there was no indication for surgical treatment. His current condition is stable two years after our initial observation.

\section{Case 3}

The patient, a 56-year-old bricklayer, had consulted a chiropractor for neck and arm pain. He reported that, 


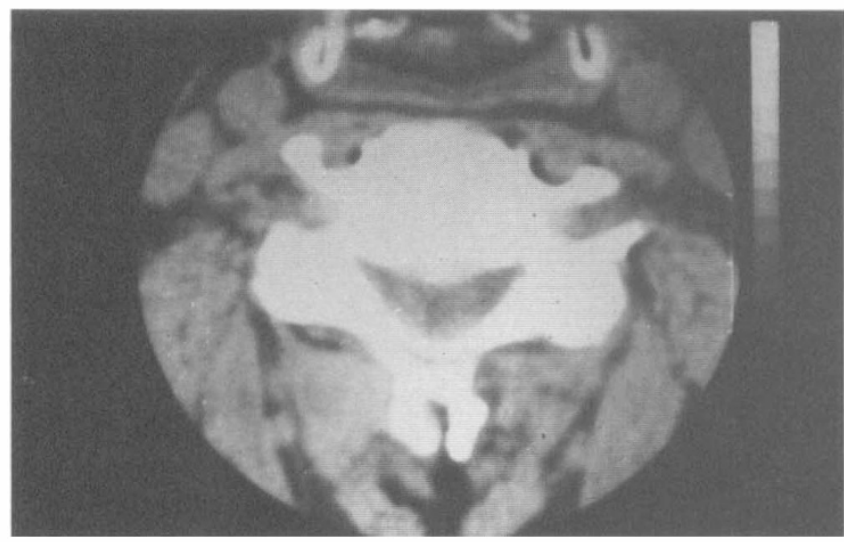

Figure 2 Case 2: Axial CT at the C4-C5 level performed after spinal manipulation. Central protrusion of the disc has obliterated the subarachnoid space and is exerting pressure on the dural sac

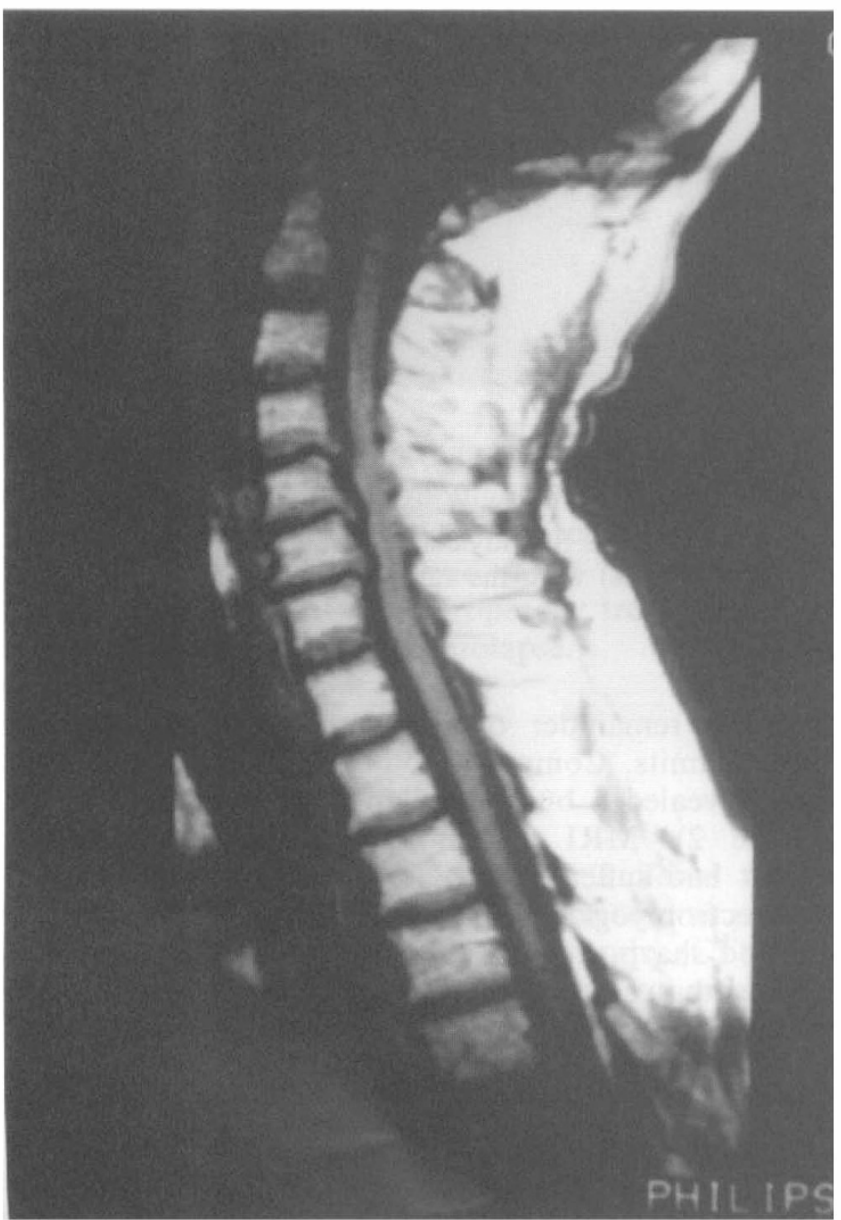

Figure 3 Case 3: Sagittal T-1 dependent MRI: marked stenosis of the spinal canal due to spondylosis and protrusion of the $\mathrm{C} 4-\mathrm{C} 5, \mathrm{C} 5-\mathrm{C} 6$ and $\mathrm{C} 6-\mathrm{C} 7$ discs, which are compressing the ventral surface of the dural sac immediately after the first cervical manipulation, he felt 'an electric shock' pass through his trunk and limbs when he flexed his neck (Lhermitte's sign). He was unable to walk and movement of the upper limbs, particularly the left, was extremely difficult.

When seen by our staff 3 weeks later, he had sensory and motor deficits of the upper limbs, more severe in the

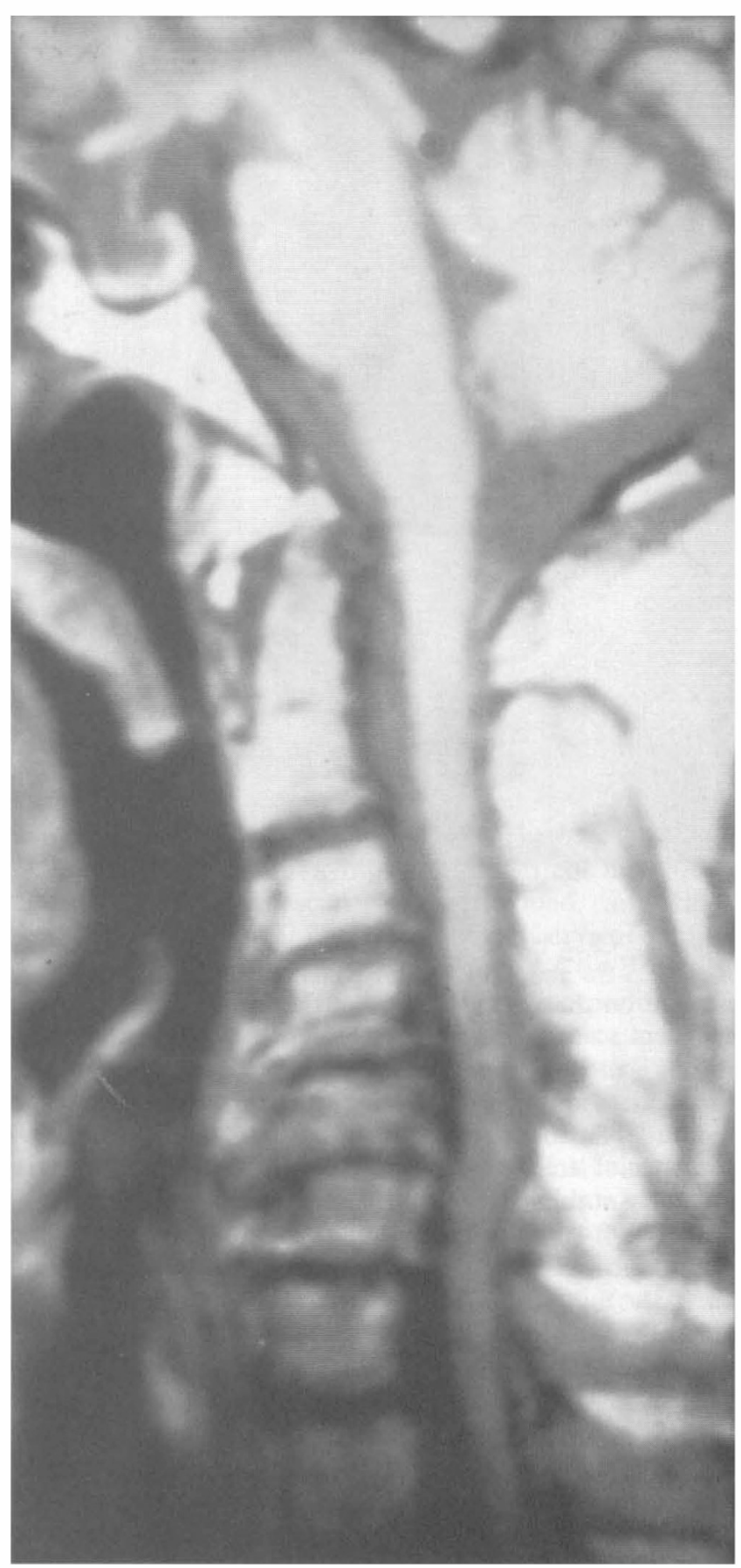

Figure 4 Case 4: Sagittal T-1 dependent MRI: extensive degenerative changes from $\mathrm{C} 3$ to $\mathrm{C} 7$. Spondylosis at $\mathrm{C} 5-\mathrm{C} 6$ level results in pressure on the ventral surface of the spinal cord 
left arm in general and there was particular involvement of the left extensor muscles. Examination of the lower limbs revealed decreased deep sensation, and, loss of postural sense. There were Babinski signs.

$\mathrm{X}$-rays of the cervical spine taken on admission to hospital showed diffuse spondyloarthrosis. On MRI, the cervical canal was seen to be stenotic, in part due to protrusion of the $\mathrm{C} 4-\mathrm{C} 5, \mathrm{C} 5-\mathrm{C} 6$ and $\mathrm{C} 6-\mathrm{C} 7$ discs, without obvious involvement of the spinal cord (Figure 3). Cervical myelopathy was confirmed by somatosensory evoked potential studies, and by cortical magnetic stimulation studies.

An operation, consisting of a C3-C6 'open-door' laminectomy was performed, and follow-up examination 1 month later revealed objective improvement. The patient was able to walk with the aid of crutches, although his gait was markedly ataxic. He was lost to follow-up shortly after this visit.

\section{Case 4}

This 62-year-old brick-layer had experienced cervical pain and bilateral hand dysesthesiae for approximately 1 year. During the last 3 months had also noted that his legs 'felt strange' when he walked. He consulted a chiropractor who prescribed manipulation of the cerivical spine. Immediately after the second manipulation, he developed weakness of all four limbs and was only able to walk with great difficulty. These symptoms were accompanied by sensory deficits of the trunk and of the upper and lower limbs.

When the patient was admitted to our department 1 month later neurological examination revealed hyposthenia of all four limbs, markedly polykinetic reflexes in the legs, an increased right triceps reflex, bilateral ankle clonus, hypoesthesia caudal to the transmamillary line and bilateral absence of position sense in the great toes. Mental status and cranial nerve functions were normal.

MRI performed after admission to hospital showed stenosis of the spinal canal between $\mathrm{C} 3$ and $\mathrm{C} 7$, particularly severe at the C5-C6 level (Figure 4). The spinal cord was clearly compressed and impairment was evident. The cervical myelopathy was confirmed with somatosensory evoked potential and cortical magnetic stimulation studies.

A bilateral C5-C7 decompressive laminectomy was performed, and there was rapid improvement of all motor deficits. Two years after surgery, the patients is able to stand and to walk with the aid of a crutch.

\section{Discussion}

Flexion and extension movements of the cervical spine involve sliding movements of one vertebra over the one below it. This movement is related to the posteroinferior orientation of the articular facets, and even normal flexion or extension reduces the anteroposterior diameter of the cervical canal. ${ }^{10-12}$

Spinal manipulation has been defined by Maigne as passive and forced movements which bring the constituent elements of one or more articulations beyond their physiological excursion. ${ }^{13}$ In patients with stenosis of the spinal canal or vertebral instability, a combination of forced rotatory and extension maneuvers during cervical spine manipulation can lead to an even more extreme excursion of the vertebrae, which may result in direct contusion ${ }^{3}$ and/ or ischemia of the spinal cord.

Pre-existing vertebral instability was documented in Case 1 and was probably also present in Case 2 . Accentuation of the vertebral movements during manipulation caused by this condition probably placed excessive stress on the anulus fibrosus which led to herniation of the discs. In Case 1, MRI scans were performed before and after the manipulation associated with the onset of radicular symptoms. It should be noted that the patient had not experienced any aggravation of symptoms prior to this treatment. Comparison of the pre- and post-treatment MRIs, together with the patient's history, suggests that the spinal manipulation performed by the new chiropractor provoked the $\mathrm{C} 5-\mathrm{C} 6$ and $\mathrm{C} 6-\mathrm{C} 7$ intervertebral disc prolapse seen in the post-treatment scan. The nerveroot damage documented by EMG was probably caused in part by protrusion of the discs. However, the multiradicular nature of the damage, and particularly the presence of the post-ganglionic damage to the C7 nerve root, suggests that it may also have been the result of mechanical stretching of the roots. ${ }^{8,14}$

The patients reported here, along with some others present in the literature, ${ }^{3-9}$ confirm that cervical manipulation can cause severe spinal cord and/or radicular damage in patients with pre-existing vertebral pathology, such as canal stenosis or vertebral instability and that old age also predisposes to neurological complications. In order to avoid severe and irreversible neurological damage, patients being considered for this type of treatment should always be subjected to neurological and orthopedic (locomotor system) examinations, including standard and dynamic $\mathrm{X}$-rays of the cervical spine. If the presence of myelopathy and stenosis or instability cannot be excluded with certainty on the basis of these results, MRI and/or evoked potential studies should be performed. This type of screening can reveal subclinical pathology of the vertebral column or spinal cord with an absolute contraindication for spinal manipulation.

\section{References}

1 Krueger B and Okazaki H. Vertebral basilar infarction following chiropractic cervical manipulation. Majo Clin Proc 1980; 55: $322-332$

2 Schellhas KP, Latchaw RE, Wendling LR and Gold LHA. Vertebrobasilar injuries following cervical manipulation. JAMA 1980; 244: $1450-1453$.

3 Schmidley JW and Koch T. The noncerebrovascular complications of chiropractic manipulation. Neurology (N.Y.) 1984; 34: $684-685$. 
4 Davis C. Osteopathic manipulation resulting in damage to spinal cord. Br Med J 1985; 291: 1540 - 1541 .

5 Shafrir Y and Kaufman BA. Quadriplegia after chiropractic manipulation in an infant with congenital torticollis caused by a spinal cord astrocitoma. J Pediatrics 1992; 120: $266-272$.

6 Rinsky LA, Reynolds GG, Jameson RM and Hamilton RD. A cervical spinal cord injury following chiropractic manipulation. Paraplegia 1976; 13: 223-227.

7 Lewis $M$ and Grundy D. Vertebral osteomyelitis following manipulation of spondylitic necks - a possible risk. 1992; 30: $788-790$.

8 Togle C, Iyer V and McConnell J. Phrenic nerve palsy accompanying chiropractic manipulation of the neck. Southern Med J 1993; 86: 688-690.

9 Pratt-Thomas HR and Berger K. Cerebellar and spinal injuries after chiropractic manipulation. JAMA 1947; 133: 600-603.
10 White AA III and Panjabi MM. Clinical Biomechanics of the Spine, 2nd ed. Philadelphia: J.B. Lippincott Co. 1990.

11 Becher M, Front D, Bornstein B and Matz S. Cervical myelopathy caused by narrowing of the cervical spinal canal: the value of $x$-ray examination of cervical spine column in extension. Clin Radiol 1971; 22: 63-68.

12 Penning L. Some aspects of plain radiography of the cervical spine in chronic myelopathy. Neurology 1962; 12: 513-519.

13 Maigne R. Douleurs d'origine vertebrale et traitments par manipulation. Paris: L'expansion 1972.

14 Breig A. Adverse mechanical tension in the central nervous system: relief by functional neurosurgery. Stockholm: Almsqvist and Wiksell International 1978. 